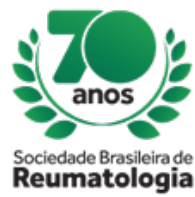

\title{
DIFFERENT RESPONSES OF FIBROBLAST-LIKE SYNOVIOCYTES FROM PATIENTS WITH RHEUMATOID ARTHRITIS AND FROM MICE WITH COLLAGEN-INDUCED ARTHRITIS TO INFLAMMATORY STIMULI
}

Camilla Ribeiro Lima Machado (Programa de Pós Graduação em Ciências Aplicadas à Saúde do Adulto, Universidade Federal de Minas Gerais, Belo Horizonte, MG, Brasil), Gustavo Gomes Resende (Hospital das Clínicas da Universidade Federal de Minas Gerais, Empresa Brasileira de Serviços Hospitalares, Belo Horizonte, MG, Brasil), Tamiris Paula Silva (Programa de Pós Graduação em Ciências Aplicadas à Saúde do Adulto, Universidade Federal de Minas Gerais, Belo Horizonte, MG, Brasil), Suelen Pizzolatto Dalmolin (Hospital de Clínicas de Porto Alegre, Serviço de Reumatologia, Universidade Federal do Rio Grande do Sul, Porto Alegre, RS, Brasil), Mirian Farinon (Hospital de Clínicas de Porto Alegre, Serviço de Reumatologia, Universidade Federal do Rio Grande do Sul, Porto Alegre, RS, Brasil), Ricardo Machado Xavier (Hospital de Clínicas de Porto Alegre, Serviço de Reumatologia, Universidade Federal do Rio Grande do Sul, Porto Alegre, RS, Brasil), Adriana Maria Kakehasi (Programa de Pós Graduação em Ciências Aplicadas à Saúde do Adulto, Universidade Federal de Minas Gerais, Belo Horizonte, MG, Brasil), Marcus Vinicius Melo Andrade (Programa de Pós Graduação em Ciências Aplicadas à Saúde do Adulto, Universidade Federal de Minas Gerais, Belo Horizonte, MG, Brasil)

\section{BACKGROUND}

Rheumatoid arthritis (RA) is a chronic autoimmune systemic disease and its main pathophysiological site is the synovial joint. Fibroblast-like synoviocytes (FLS) actively participate in the inflammation-damage cycle, through the production of cytokines such as interleukin-6 (IL-6) and cartilage-degrading enzymes, such as metalloproteinases (MMPs). Tumor necrosis factor alpha (TNF- $\alpha$ ) and interleukin-1beta (IL-1 $\beta$ ) are overexpressed and play important roles in its pathogenesis. Murine FLS from experimental animal models of arthritis are used as substitutes for human cells in in vitro assays, however, little has been found about functionality comparisons between FLS obtained from humans with RA and from mice. The aim of this study is to compare the production of IL-6 and MMP-3 and the activation of intracellular signaling pathways (NF-KB and MAPK: p-ERK1/2, p-P38 and p-JNK) in FLS cultures obtained from patients with RA (RA-FLS) and mice with collagen-induced arthritis (CIA-FLS).

\section{MATERIALS AND METHODS}

RAFLS $(n=8)$ and CIA-FLS $(n=4)$ were stimulated with $50 \mathrm{ng} / \mathrm{ml}$ TNF- $\alpha$ and $1 \mathrm{ng} / \mathrm{ml} \mathrm{IL-1 \beta}$. After 24 hours of stimulation, IL- 6 and MMP-3 were dosed in the supernatant by ELISA. In the cell lysate, activation of MAPK signaling pathways ( $\mathrm{p}-\mathrm{ERK} 1 / 2, \mathrm{p}-\mathrm{P} 38$ and $\mathrm{p}-\mathrm{JNK}$ ) and NF-KB was assessed by Western Blot.

\section{RESULTS}

Both RA-FLS and CIA-FLS constitutively produce MMP-3 and IL-6 (present even without stimulation). Increased production of IL- 6 and MMP-3 was observed in RA-FLS after TNF- $\alpha$ and IL-1 $\beta$ stimulation, all of them with statistical significance ( $p=0.0267, p=0.0405, p=0.0405$ and $p=0.0075$, respectively). In CIAFLS, however, TNF- $\alpha$ stimulation increases only IL-6 production $(p=0.0026)$. No difference was found in the production of MMP-3 after TNF- $\alpha$, neither in IL- 6 and MMP-3 after IL-1 $\beta$ stimulation. Similarly, regarding activation of the signaling pathways studied, TNF- $\alpha$ activated $p$-ERK1/2 in both groups, but $p$ JNK only in humans and p-P38 only in mice. IL-1 $\beta$ activated p-ERK1/2, p-P38 and NF-KB in humans, but in mice only p-P38 activated discretely and the rest of the pathways (p-ERK1/2, p-JNK and NF-KB) were not activated.

\section{CONCLUSION}


This study demonstrated significantly differences in murine and human FLS responses to inflammatory stimuli, both at protein production level and activation of signaling pathways, which does not support the replacement of RA-FLS by CIA-FLS in experiments that plan to study the behavior of FLS in RA. 